\section{Fatores associados ao baixo conhecimento sobre HIVIAIDS entre homens que fazem sexo com homens no Brasil}

\author{
Factors associated with low knowledge on \\ HIV/AIDS among men who have sex with \\ men in Brazil
}

\section{Factores asociados al bajo conocimiento sobre VIH/SIDA entre hombres que practican sexo con hombres en Brasil}

\section{Resumo}

Este estudo transversal analisou os fatores de vulnerabilidade social, individual e programática associados ao baixo conhecimento em HIV/AIDS entre homens que fazem sexo com homens (HSH). A técnica amostral Respondent Driven Sampling (RDS) foi utilizada em dez cidades brasileiras. Os escores de conhecimento em HIV/AIDS foram estimados pela Teoria de Resposta ao Item e categorizados em três níveis: alto, médio e baixo conhecimento. $\mathrm{O}$ modelo de regressão logística ordinal foi utilizado para a análise. Dentre os 3.746 HSH, encontrou-se 36,6\%, 37,4\% e 26\% com alto, médio e baixo conhecimento, respectivamente. No modelo final, apresentaram associação com o baixo conhecimento: escolaridade $\leq 8$ anos, cor da pele não branca, critério de classe econômica $C$ e $D$-E, idade $<25$ anos, ter somente um parceiro sexual e nunca ter feito o teste anti-HIV. É fundamental melhorar o nível de conhecimento sobre HIV/AIDS entre os jovens HSH, com condições socioeconômicas desfavoráveis. Intervenções focadas nos fatores de vulnerabilidade programática podem contribuir para a redução dos fatores individual e social.

HIV; Grupos de Risco; Homossexualidade Masculina; Conhecimentos, Atitudes e Prática em Saúde; Vulnerabilidade em Saúde
Raquel Regina de Freitas Magalhães Gomes 1,2 Maria das Graças Braga Ceccato 3 Lígia Regina Franco Sansigolo Kerr 4 Mark Drew Crosland Guimarães 1

doi: 10.1590/0102-311X00125515
Correspondência

R. R. F. M. Gomes

Rua Dr. Virgílio Uchoa 627, Belo Horizonte, MG

30320-240, Brasil.

quelfmg@gmail.com

1 Faculdade de Medicina, Universidade Federal de Minas Gerais, Belo Horizonte, Brasil.

2 Secretaria Municipal de Saúde de Belo Horizonte, Belo Horizonte, Brasil

3 Faculdade de Farmácia, Universidade Federal de Minas Gerais, Belo Horizonte, Brasil.

4 Faculdade de Medicina, Universidade Federal do Ceará, Fortaleza, Brasil. 


\section{Introdução}

A epidemia do vírus da imunodeficiência humana (HIV) em homens que fazem sexo com homens (HSH) continua a se expandir na maioria dos países. Em países como os Estados Unidos, observase uma estabilização da epidemia nessa população, ainda que com diferenças importantes por faixa etária e raça/cor ${ }^{1}$. A alta probabilidade de transmissão por ato sexual, principalmente por meio da relação anal receptiva desprotegida, tem um papel central na explicação da carga da doença desproporcional nessa população 2. Estudo nacional realizado entre HSH de dez municípios brasileiros, utilizando a metodologia Respondent Driven Sampling (RDS), mostrou uma preocupante situação epidemiológica. A prevalência global do HIV foi de 14,2\% 3 com variações de 5,2 a 23,7\% entre as cidades, e a proporção de relações anais receptivas desprotegidas foi de 36,5\% 4. Da mesma forma, o nível de conhecimento em HIV/AIDS entre os HSH foi avaliado e encontrou-se 40,7\% da amostra com níveis de conhecimento abaixo da média 5 .

Em geral, modelos teóricos que buscam compreender mudanças comportamentais em populações em maior risco para o HIV e outras doenças sexualmente transmissíveis (DST) se fundamentam na suposição de que prover conhecimento básico sobre o HIV/AIDS potencialmente conduz à mudança de comportamento. O acesso à informação e educação de massa são componentes fundamentais em programas que visem a aumentar a conscientização sobre os modos de transmissão e prevenção do HIV/AIDS, acalmando as ansiedades acerca da transmissão e prevenindo a discriminação. Contudo, é de consenso que não se pode subestimar a relação interativa das dimensões sociocultural, política e econômica na transformação do conhecimento em comportamento sexual mais seguro ${ }^{6}$. A diversidade de contextos interfere nesse processo, diferenciando os indivíduos com relação ao seu conhecimento sobre o HIV e seu comportamento. Sendo assim, esforços são necessários para lidar com os determinantes mais amplos de comportamento sexual, especialmente aqueles que se relacionam com o contexto social. Identificar as diferenças e suas especificidades é imprescindível no planejamento e implantação de políticas e programas voltados para o atendimento de grupos mais vulneráveis à exposição ao HIV, dentre estes, a população de HSH 7.

Considerando de fundamental importância a compreensão dos fatores relacionados ao conhecimento em HIV entre os HSH, este estudo utilizou como base o referencial teórico de vulnerabilidade proposto por Mann et al. 8 . No início da epidemia, esses autores introduziram o conceito de vulnerabilidade e desenvolveram uma estrutura de análise para avaliar o grau de vulnerabilidade do indivíduo ou de um determinado grupo à infecção pelo HIV, relacionado a um conjunto de fatores que podem aumentar ou diminuir o risco de adoecimento. Esses conceitos foram ampliados e utilizados por pesquisadores para avaliar e compreender a evolução da doença em populações específicas 9,10,11,12.

Segundo os autores, a vulnerabilidade à infecção pelo HIV/AIDS incorpora três dimensões que interagem de forma dinâmica e interdependentes: (1) vulnerabilidade social foca diretamente os fatores contextuais, o acesso à informação, saúde, educação, o poder de enfrentar barreiras culturais e de influenciar decisões políticas; (2) vulnerabilidade individual diz respeito às dimensões cognitivas e comportamentais, ao grau e à qualidade da informação que os indivíduos dispõem sobre os problemas de saúde, suas atitudes e práticas; (3) vulnerabilidade programática é referente ao grau de comprometimento das políticas governamentais e não governamentais com o enfrentamento da AIDS, aos investimentos e financiamentos em ações assistenciais e preventivas, ao monitoramento dos programas e sustentabilidade destas ações 9 .

No Brasil, são escassos os trabalhos que avaliaram o conhecimento sobre HIV, sendo os mais abrangentes os inquéritos nacionais que ocorreram em 2004 e 2008 na população geral brasileira 13,14. Estudos realizados em outros países avaliaram o conhecimento sobre HIV, especificamente entre os $\mathrm{HSH}$, e encontraram resultados relevantes que mostraram os seguintes fatores associados com o baixo conhecimento sobre HIV: menor escolaridade; idade menor que 25 anos e maior do que 50; grupo racial não branco; nunca ter realizado teste para HIV; HSH com resultado negativo para HIV; não usar preservativos na última relação sexual e menor número de parceiros sexuais 15,16,17,18,19.

Sendo assim, o objetivo deste estudo foi avaliar e identificar os fatores indicadores de vulnerabilidade social, individual e programática associados ao baixo conhecimento sobre HIV/AIDS em uma amostra da população de HSH recrutados em dez cidades brasileiras. 


\section{Metodologia}

\section{Desenho}

Esta análise faz parte de um estudo nacional, de corte transversal, realizado em 2008-2009 com HSH residentes em dez cidades brasileiras. Teve, entre seus objetivos, estimar a prevalência da infecção por HIV e sífilis e avaliar o conhecimento em HIV/AIDS, atitudes e práticas sexuais na população de HSH. As cidades selecionadas foram Manaus (Amazonas), Brasília (Distrito Federal), Recife (Pernambuco), Salvador (Bahia), Campo Grande (Mato Grosso do Sul), Belo Horizonte (Minas Gerais), Rio de Janeiro, Santos (São Paulo), Curitiba (Paraná) e Itajaí (Santa Catarina). O estudo foi aprovado pelo Comitê Nacional de Ética em Pesquisa (CONEP no 14494) 3.

\section{Recrutamento e população do estudo}

Uma amostra de 250 a $350 \mathrm{HSH}$ foi estimada por cidade e recrutada utilizando-se o método de amostragem por cadeia, Respondent Driven Sampling (RDS), recomendado para populações de difícil acesso 3,20. Foram considerados elegíveis os participantes com idade igual ou superior a 18 anos, residentes em uma das cidades selecionadas, que tiveram pelo menos uma relação sexual com um homem nos últimos 12 meses, apresentaram um cupom de participação válido e assinaram o Termo de Consentimento Livre e Esclarecido. Por meio de pesquisa formativa (grupos focais e entrevistas), os primeiros participantes, denominados de sementes, foram selecionados de membros da populaçãoalvo para cada cidade. Neste estudo, cinco sementes foram selecionadas para cada cidade, que após consentirem em participar da pesquisa, receberam três cupons numerados para convidar pessoas da sua rede social. Os primeiros recrutados que chegaram ao local da pesquisa com um cupom válido e que cumpriram com os critérios de inclusão, constituíram a primeira onda. Esse processo foi se repetindo até que o estudo atingisse o tamanho amostral desejado. Os participantes receberam duplo incentivo financeiro, pela sua participação no estudo e por cada um dos seus recrutados que apresentaram um cupom de participação. Os dados sobre as características socioeconômicas e demográficas, rede social, conhecimento sobre HIV/AIDS, atitudes e práticas sexuais foram coletados por meio de entrevista estruturada face a face mediada por um computador de bolso (pocket-PC). Detalhes metodológicos do estudo estão disponíveis em Kerr et al. ${ }^{3}$.

\section{Evento e variáveis explicativas}

O evento considerado para análise foi o conhecimento sobre HIV/AIDS medido baseando-se em dez afirmativas contidas no questionário sobre formas de transmissão, das quais cinco itens foram recomendados pela Sessão Extraordinária da Assembleia Geral das Nações Unidas (UNGASS) 21, para medir o conhecimento em HIV/AIDS em subgrupos de risco: (1) o risco de transmissão do HIV pode ser reduzido se houver relações sexuais somente com um parceiro não infectado que não tenha outros parceiros; (2) uma pessoa pode pegar o vírus da AIDS se não usar preservativos; (3) uma pessoa saudável pode estar infectada pelo vírus da AIDS; (4) uma pessoa pode pegar o vírus da AIDS se for picada por insetos; (5) uma pessoa pode se infectar ao compartilhar talheres, copos ou alimentos. As outras cinco questões foram propostas pelo Ministério da Saúde: (6) o risco de transmissão do HIV é pequeno se a pessoa fizer o tratamento corretamente; (7) as pessoas estão usando menos preservativo por causa do tratamento para a AIDS; (8) uma pessoa pode pegar o vírus da AIDS se usar banheiros públicos; (9) o risco da mãe HIV positiva infectar seu bebê é menor se ela recebe tratamento durante a gravidez e o parto; (10) uma pessoa pode pegar o vírus da AIDS se compartilhar uma seringa ou agulha.

O conjunto dos itens do questionário apresentava três opções de respostas: sim (concorda), não (discorda) e não sabe. As respostas corretas foram categorizadas como "um" e as incorretas e não sabe como "zero". Os escores de conhecimento foram estimados pelo modelo logístico de dois parâmetros (dificuldade e discriminação) fundamentado na Teoria de Resposta ao Item (TRI), cuja metodologia está detalhada no estudo de Gomes et al. 5. 
Neste trabalho, os escores de conhecimento foram categorizados como uma variável ordinal em três níveis ordenados: alto, médio e baixo conhecimento.

As variáveis explicativas selecionadas foram agrupadas segundo as três dimensões de vulnerabilidade:

(1) Vulnerabilidade social: escolaridade; cor da pele; estado conjugal; com quem mora; trabalha atualmente; renda individual; critério Brasil de classe econômica; discriminação e história de agressão verbal devido à orientação sexual nos últimos 12 meses; contou para alguém que tem atração sexual por homens; amigos conversam sobre prevenção ou incentivam o uso de preservativos.

(2) Vulnerabilidade individual: idade; idade da primeira relação sexual; orientação sexual; número de parceiros sexuais; utilizou lugares ou serviços para encontrar parceiros sexuais; uso de preservativo em relação anal receptiva com qualquer tipo de parceria; chances de se infectar com o HIV; frequência de bebidas alcoólicas; uso abusivo de álcool (binge); esquecer-se de usar preservativos com uso de álcool e drogas; relação sexual com ingestão de bebida alcoólica e com uso de droga ilícita; história de DST; sentir-se triste ou deprimido; resultado de HIV e de sífilis.

(3) Vulnerabilidade programática: participa de atividade religiosa, de atividades em serviços de saúde; conhece e participa de algum grupo organizado ou organização não-governamental (ONG); fez teste para HIV e sífilis; recebeu preservativos gratuitos e gel lubrificante.

\section{Análise estatística}

Neste estudo, os dez municípios foram considerados simultaneamente. A amostra foi ponderada com base no inverso da probabilidade de seleção individual, proporcional ao tamanho da rede social informada por cada participante e pela proporção de HSH para cada cidade em relação ao total amostral 3,22.

Realizou-se uma análise descritiva das variáveis explicativas por meio de distribuição de frequência das variáveis categóricas e medidas de tendência central das variáveis contínuas. A definição do ponto de corte da variável resposta ordinal (conhecimento) foi baseada em análise por meio da teoria da resposta ao item publicado anteriormente 5 . Dessa forma, os valores dos percentis 75 e 25 foram considerados para o agrupamento do grau de conhecimento em três categorias (k) de conhecimento: alto (> 75), médio (75-25) e baixo $(<25)$.

Foi utilizado o modelo de regressão logístico ordinal, adequado para análise de dados ordinais, quando a variável resposta é ordenada baseando-se em uma variável contínua agrupada. Esse modelo é também denominado de odds proporcional, no qual (k-1) regressões logísticas são ajustadas simultaneamente, gerando uma medida de associação constante ao longo das categorias da variável resposta 23,24. Desse modo, trabalhou-se no ajuste de um modelo logístico odds proporcional ponderado, avaliando o efeito independente das potenciais variáveis explicativas em relação às categorias de comparação.

Inicialmente, foi realizada uma análise univariada considerando-se a natureza ordenada da variável resposta. As diferenças de proporção foram avaliadas pelo teste do qui-quadrado de tendência linear, com nível de significância de 0,05. A magnitude das associações foi estimada pelo cumulative odds ratio (OR), ou seja, pela probabilidade cumulativa entre as duas categorias de menor conhecimento, com intervalo de 95\% de confiança (IC95\%). Posteriormente, foram produzidos modelos intermediários, um para cada grupo de vulnerabilidade, incluindo todas as variáveis da análise univariada com nível de significância valor de $\mathrm{p}<0,20$. A deleção sequencial foi realizada de acordo com a importância estatística de cada variável até se alcançar o ajuste final de cada modelo intermediário separadamente, considerando valor de $\mathrm{p}<0,05$ e o escore do teste para o pressuposto do odds proporcional. $\mathrm{Na}$ modelagem final foram incluídas todas as variáveis dos três modelos intermediários e considerou-se para ajuste do modelo os mesmos critérios dos modelos intermediários, valor de $\mathrm{p}<0,05$ e o escore do teste para odds proporcional. O software SAS 9.0 (SAS Inst., Cary, Estados Unidos) foi usado para a análise estatística dos dados. 


\section{Resultados}

Dos 3.746 participantes do estudo, 1.370 HSH (36,6\%) foram categorizados como alto conhecimento, $1.401 \mathrm{HSH}(37,4 \%)$ como médio conhecimento e $975 \mathrm{HSH}$ (26\%) como baixo conhecimento (Tabela 1). As variáveis indicadoras de vulnerabilidade social mostraram que a maioria dos HSH tinha mais de oito anos de estudo (58,3\%), pele não branca (74\%), eram solteiros $(84,8 \%)$, com trabalho $(66,5 \%)$, com renda individual < R\$ 750,00 (74,3\%), que não se sentiram discriminados $(72,4 \%)$ e não sofreram agressão verbal (56,6\%) nos últimos 12 meses devido à sua orientação sexual, e que já tinham revelado sua identidade sexual para alguém do grupo familiar e/ou social (74\%).

Quanto às variáveis indicadoras de vulnerabilidade individual, a população estudada tinha 25 anos ou mais $(62,3 \%)$ e os HSH referiram a primeira relação sexual com mais de 14 anos (53,4\%). Aproximadamente $32 \%$ relataram fazer uso inconsistente de preservativos em relação anal receptiva nos últimos seis meses, e 44,3\% declararam já ter se esquecido de usar preservativos com o uso de álcool e drogas. Uma proporção considerável de HSH relatou consumir bebidas alcoólicas duas ou mais vezes por semana $(63,7 \%)$ e ter relação sexual após a ingestão $(61,3 \%)$.

Dentre as variáveis indicadoras de vulnerabilidade programática, destaca-se a alta proporção de HSH que nunca fez o teste para o HIV (49,5\%) e para a sífilis (75\%). Com relação aos insumos, 85,1\% nunca receberam gel lubrificante nos últimos 12 meses e 44,1\% dos HSH relataram nunca ter recebido preservativos gratuitos, e os que receberam informaram que foram insuficientes.

A análise univariada (Tabela 1) indicou que a maioria das variáveis indicadoras dos três grupos de vulnerabilidade apresentou associação estatisticamente significativa com o baixo conhecimento. As análises multivariadas intermediárias (Tabela 2) indicaram associações estatisticamente significativas com o baixo conhecimento para as seguintes variáveis de cada grupo:

(1) Vulnerabilidade social: escolaridade $\leq 8$ anos; cor da pele não branca; morar com os pais; renda individual < R\$ 750,00; critério Brasil de classe econômica: C e D-E.

(2) Vulnerabilidade individual: idade < 25 anos; idade da 1a relação sexual $\leq 14$ anos; orientação sexual: HSH/homossexual e bissexual/outros; ter somente um parceiro sexual nos últimos seis meses; não sabe a sua chance de se infectar com o vírus do HIV; esquecer de usar preservativos com o uso de álcool e drogas; não ter relação sexual com o uso de álcool e drogas nos últimos 6 meses; ter relação sexual com o uso de drogas ilícitas nos últimos seis meses.

(3) Vulnerabilidade programática: não conhecer algum grupo organizado ou ONG; nunca ter feito o teste de HIV; nunca ter feito o teste de sífilis; não recebeu ou recebeu quantidade insuficiente de preservativos gratuitos no último mês; não recebeu gel lubrificante nos últimos 12 meses.

No modelo final global, estiveram associadas de forma independente com as categorias de baixo conhecimento as seguintes variáveis: escolaridade $\leq 8$ anos $(\mathrm{OR}=2,11)$, cor da pele não branca $(\mathrm{OR}=1,32)$, critério Brasil de classe econômica $\mathrm{C}(\mathrm{OR}=1,62)$ e $\mathrm{D}-\mathrm{E}(\mathrm{OR}=2,10)$, idade $<25$ anos $(\mathrm{OR}=1,78)$, ter somente um parceiro sexual nos últimos seis meses $(\mathrm{OR}=1,30)$ e nunca ter feito o teste anti-HIV $(\mathrm{OR}=2,72)$. Os resultados dos escores dos testes dos modelos intermediários e final ( $\mathrm{p}>0,05$ ), mostrados na Tabela 2, validaram o pressuposto do odds proporcional, indicando que os coeficientes (ß) estimados eram idênticos entre as categorias de comparação cumulativas do baixo conhecimento.

\section{Discussão}

Os resultados obtidos neste trabalho indicam elevada proporção de médio ou baixo nível de conhecimento sobre as formas de transmissão da infecção entre a população de HSH no Brasil (63,4\%). Torna-se ainda mais preocupante se considerarmos que os itens avaliados abordam informações básicas amplamente divulgadas. A utilização da metodologia TRI permitiu avaliar não somente o grau de conhecimento, mas também os itens considerados mais difíceis e aqueles com a melhor capacidade de discriminação 5,25 .

As associações encontradas dos fatores indicadores de vulnerabilidade social com o baixo conhecimento evidenciam a desigualdade social encontrada no país, um indicador agravante na disseminação da infecção pelo HIV 26. O contexto social da epidemia do HIV/AIDS entre os estratos populacionais 
Tabela 1

Análise univariada dos níveis de conhecimento em HIV/AIDS segundo os fatores de vulnerabilidade entre os homens que fazem sexo com homens $(\mathrm{HSH})$, Brasil $(\mathrm{N}=3.746)$.

\begin{tabular}{|c|c|c|c|c|c|c|}
\hline \multirow[t]{3}{*}{ Fatores de vulnerabilidade } & \multicolumn{4}{|c|}{ Níveis de conhecimento * } & \multirow[t]{3}{*}{ OR ** (IC95\%) } & \multirow[t]{3}{*}{ Valor de $p$} \\
\hline & Total & $\begin{array}{c}\text { Alto } \\
(n=1.370)\end{array}$ & $\begin{array}{c}\text { Médio } \\
\text { (n=1.401) }\end{array}$ & $\begin{array}{c}\text { Baixo } \\
(n=975)\end{array}$ & & \\
\hline & $\%$ & n (\%) & n (\%) & n (\%) & & \\
\hline \multicolumn{7}{|l|}{ Social } \\
\hline \multicolumn{7}{|l|}{ Escolaridade (anos) } \\
\hline $12+$ & 27,4 & $634(49,9)$ & $487(38,4)$ & $149(11,7)$ & 1,00 & \\
\hline $9-11$ & 30,9 & $471(35,2)$ & $527(39,5)$ & $338(25,3)$ & $2,08(1,77-2,43)$ & $<0,0001$ \\
\hline$\leq 8$ & 41,7 & $257(22,9)$ & $382(34,0)$ & $485(43,1)$ & $4,79(4,11-5,59)$ & $<0,0001$ \\
\hline \multicolumn{7}{|l|}{ Cor da pele } \\
\hline Branca & 26,0 & $452(43,7)$ & $372(36,0)$ & $210(20,3)$ & 1,00 & \\
\hline Não branca & 74,0 & $915(33,8)$ & $1.029(38,0)$ & $765(28,2)$ & $1,96(1,71-2,25)$ & $<0,0001$ \\
\hline \multicolumn{7}{|l|}{ Estado conjugal } \\
\hline Solteiro & 84,8 & $1.143(36,6)$ & $1.166(37,3)$ & $817(26,1)$ & 1,00 & 0,43 \\
\hline Casado/União & 15,2 & $225(36,4)$ & $235(38,0)$ & $158(25,6)$ & $1,07(0,91-1,26)$ & \\
\hline \multicolumn{7}{|l|}{ Com quem mora } \\
\hline Sozinho & 22,7 & $293(42,6)$ & $250(36,3)$ & $145(21,1)$ & 1,00 & \\
\hline Parceiro/Outros & 41,2 & $494(37,4)$ & $499(37,8)$ & $329(24,9)$ & $1,10(0,94-1,30)$ & 0,25 \\
\hline Pais & 36,1 & $549(34,6)$ & $597(37,6)$ & $443(27,9)$ & $1,28(1,08-1,51)$ & 0,004 \\
\hline \multicolumn{7}{|l|}{ Trabalha atualmente } \\
\hline Sim & 66,5 & $966(40,4)$ & $887(37,1)$ & $538(22,5)$ & 1,00 & \\
\hline Não & 33,5 & $369(30,6)$ & $459(38,0)$ & $379(31,4)$ & $1,25(1,10-1,43)$ & 0,0009 \\
\hline \multicolumn{7}{|l|}{ Renda individual $(\mathrm{R} \$)$} \\
\hline$>1.299,00$ & 11,2 & $270(59,2)$ & $143(31,4)$ & $43(9,4)$ & 1,00 & \\
\hline $750,00-1.299,00$ & 14,5 & $287(45,9)$ & $251(40,1)$ & $88(14,1)$ & $1,73(1,35-2,21)$ & $<0,0001$ \\
\hline$<750,00$ & 74,3 & $812(30,5)$ & $1.004(37,8)$ & $843(31,7)$ & $4,07(3,32-4,99)$ & $<0,0001$ \\
\hline \multicolumn{7}{|c|}{ Critério Brasil de Classificação Econômica } \\
\hline$A-B$ & 24,6 & $501(48,3)$ & $385(37,1)$ & $151(14,6)$ & 1,00 & \\
\hline c & 48,6 & $658(35,3)$ & $719(38,5)$ & $488(26,2)$ & $2,48(2,11-2,91)$ & $<0,0001$ \\
\hline D-E & 26,8 & $177(25,4)$ & $242(34,8)$ & $277(39,8)$ & $3,95(3,29-4,73)$ & $<0,0001$ \\
\hline \multicolumn{7}{|c|}{ Discriminação devido à orientação sexual } \\
\hline Sim & 27,6 & $490(37,9)$ & $529(40,9)$ & $274(21,2)$ & 1,00 & \\
\hline Não & 72,4 & $879(35,9)$ & $871(35,5)$ & $700(28,6)$ & $1,31(1,15-1,49)$ & $<0,0001$ \\
\hline \multicolumn{7}{|c|}{$\begin{array}{l}\text { História da agressão verbal devido à orientação } \\
\text { sexual }\end{array}$} \\
\hline Sim & 42,4 & $685(40,3)$ & $636(37,5)$ & $377(22,2)$ & 1,00 & \\
\hline Não & 57,6 & $684(33,4)$ & $764(37,4)$ & $598(29,2)$ & $1,47(1,31-1,66)$ & $<0,0001$ \\
\hline \multicolumn{7}{|c|}{$\begin{array}{l}\text { Contou para alguém que tem atração sexual por } \\
\text { homens }\end{array}$} \\
\hline Sim & 74,0 & $1.087(39,8)$ & $1.058(38,7)$ & $588(21,5)$ & 1,00 & \\
\hline Não & 26,0 & $202(31,0)$ & $227(34,8)$ & $223(34,2)$ & $1,71(1,48-1,98)$ & $<0,0001$ \\
\hline \multicolumn{7}{|c|}{ Amigos conversam sobre prevenção } \\
\hline Todos/Maioria & 34,8 & $549(37,3)$ & $545(37,0)$ & $378(25,7)$ & 1,00 & \\
\hline Poucos/Nenhum & 65,2 & $821(36,1)$ & $854(37,6)$ & $597(26,3)$ & $1,12(0,99-1,27)$ & 0,06 \\
\hline \multicolumn{7}{|c|}{ Amigos incentivam o uso de preservativos } \\
\hline Todos/Maioria & 52,8 & $812(38,5)$ & $779(36,9)$ & $518(24,6)$ & 1,00 & \\
\hline Poucos/Nenhum & 47,2 & $558(34,1)$ & $621(38,0)$ & $457(27,9)$ & $1,09(0,97-1,23)$ & 0,15 \\
\hline
\end{tabular}

(continua) 


\begin{tabular}{|c|c|c|c|c|c|c|}
\hline \multirow[t]{3}{*}{ Fatores de vulnerabilidade } & \multicolumn{4}{|c|}{ Níveis de conhecimento * } & \multirow[t]{3}{*}{ OR ** (IC95\%) } & \multirow[t]{3}{*}{ Valor de $\mathrm{p}$} \\
\hline & Total & $\begin{array}{c}\text { Alto } \\
(n=1.370)\end{array}$ & $\begin{array}{l}\text { Médio } \\
(n=1.401)\end{array}$ & $\begin{array}{c}\text { Baixo } \\
(\mathrm{n}=975)\end{array}$ & & \\
\hline & $\%$ & n (\%) & n (\%) & n (\%) & & \\
\hline \multicolumn{7}{|l|}{ Individual } \\
\hline \multicolumn{7}{|l|}{ Idade (anos) } \\
\hline$>35$ & 27,8 & $288(42,8)$ & $243(36,2)$ & $141(21,0)$ & 1,00 & \\
\hline $25-35$ & 34,5 & $554(43,7)$ & $453(35,7)$ & $262(20,6)$ & $1,26(1,08-1,47)$ & 0,003 \\
\hline$<25$ & 37,7 & $521(29,1)$ & $700(39,1)$ & $569(31,8)$ & $1,92(1,65-2,22)$ & $<0,0001$ \\
\hline \multicolumn{7}{|l|}{ Idade da 1ạ relação sexual (anos) } \\
\hline$>18$ & 11,8 & $156(48,2)$ & $107(33,0)$ & $61(18,8)$ & 1,00 & \\
\hline $15-18$ & 41,6 & $566(36,7)$ & $565(36,7)$ & $410(26,6)$ & $1,37(1,13-1,68)$ & 0,002 \\
\hline$\leq 14$ & 46,6 & $642(34,6)$ & $721(38,8)$ & $494(26,6)$ & $1,73(1,43-2,11)$ & $<0,0001$ \\
\hline \multicolumn{7}{|l|}{ Orientação sexual } \\
\hline Gay & 17,3 & $349(43,0)$ & $327(40,3)$ & $135(16,7)$ & 1,00 & \\
\hline HSH/Homossexual & 42,4 & $596(40,2)$ & $549(37,0)$ & $337(22,7)$ & $1,24(1,05-1,47)$ & 0,01 \\
\hline Bissexual/Outro & 39,7 & $423(29,2)$ & $524(36,2)$ & $501(34,6)$ & $1,72(1,46-2,04)$ & $<0,0001$ \\
\hline \multicolumn{7}{|c|}{ Número de parceiros sexuais nos últimos 6 meses } \\
\hline+5 & 17,6 & $363(33,7)$ & $402(37,4)$ & $311(28,9)$ & 1,00 & \\
\hline $2-5$ & 37,3 & $629(39,0)$ & $589(36,5)$ & $395(24,5)$ & $0,92(0,80-1,10)$ & 0,27 \\
\hline 1 & 45,1 & $375(36,7)$ & $392(38,3)$ & $256(25,0)$ & $1,11(0,95-1,30)$ & 0,20 \\
\hline \multicolumn{7}{|c|}{$\begin{array}{l}\text { Utilizou lugares ou serviços para encontrar parceiros } \\
\text { sexuais no último mês }\end{array}$} \\
\hline Não & 58,2 & $811(37,3)$ & $821(37,7)$ & $544(25,0)$ & 1,00 & \\
\hline Sim & 41,8 & $558(35,6)$ & $579(36,9)$ & $431(27,5)$ & $1,05(0,93-1,18)$ & 0,42 \\
\hline \multicolumn{7}{|c|}{$\begin{array}{l}\text { Uso de preservativo em relação anal receptiva com } \\
\text { qualquer tipo de parceria nos últimos } 6 \text { meses }\end{array}$} \\
\hline Irregular & 31,7 & $525(39,7)$ & $511(38,7)$ & $286(21,6)$ & 1,00 & \\
\hline Sempre & 68,3 & $843(35,1)$ & $881(36,7)$ & $679(283)$ & $1,19(1,05-1,35)$ & 0,008 \\
\hline \multicolumn{7}{|c|}{ Chances de se infectar com o HIV } \\
\hline Grande/Moderada & 49,3 & $379(37,6)$ & $393(39,0)$ & $235(23,3)$ & 1,00 & \\
\hline Nenhuma/Pouca & 32,4 & $778(37,5)$ & $767(37,0)$ & $529(25,5)$ & $1,15(1,00-1,31)$ & 0,05 \\
\hline Não sabe & 18,3 & $124(24,8)$ & $176(35,2)$ & $200(40,0)$ & $1,60(1,34-1,92)$ & $<0,0001$ \\
\hline \multicolumn{7}{|c|}{ Frequência do consumo de bebidas alcoólicas } \\
\hline $2+$ vezes/semana & 63,7 & $842(37,6)$ & $814(36,4)$ & $582(26,0)$ & 1,00 & \\
\hline Nunca/Eventual & 36,3 & $527(35,0)$ & $586(39,0)$ & $391(26,0)$ & $1,25(1,10-1,41)$ & 0,004 \\
\hline \multicolumn{7}{|c|}{ Uso de álcool (binge) nos últimos 6 meses } \\
\hline Não & 61,1 & $894(37,1)$ & $931(38,6)$ & $585(24,3)$ & 1,00 & \\
\hline Sim & 38,9 & $475(35,7)$ & $469(35,2)$ & $388(29,1)$ & $1,04(0,93-1,18)$ & 0,48 \\
\hline \multicolumn{7}{|c|}{$\begin{array}{l}\text { Esquecer-se de usar preservativos com uso de álcool } \\
\text { e drogas }\end{array}$} \\
\hline Não & 55,7 & $864(38,8)$ & $814(36,5)$ & $550(24,7)$ & 1,00 & \\
\hline Sim & 44,3 & $505(33,3)$ & $587(38,7)$ & $424(28,0)$ & $1,26(1,12-1,41)$ & 0,0002 \\
\hline \multirow{2}{*}{\multicolumn{7}{|c|}{$\begin{array}{l}\text { Relação sexual com ingestão de bebida alcoólica nos } \\
\text { últimos } 6 \text { meses }\end{array}$}} \\
\hline & & & & & & \\
\hline Sim & 61,3 & $843(37,1)$ & $847(37,3)$ & $580(25,6)$ & 1,00 & \\
\hline Não & 38,7 & $526(35,8)$ & $552(37,6)$ & $391(26,6)$ & $1,27(1,13-1,43)$ & 0,0001 \\
\hline \multicolumn{7}{|c|}{ Relação sexual com uso de droga ilícita nos últimos } \\
\hline \multicolumn{7}{|l|}{6 meses } \\
\hline Não & 71,4 & $1.050(38,2)$ & $1.037(37,7)$ & $662(24,1)$ & 1,00 & \\
\hline Sim & 28,6 & $314(31,9)$ & $362(36,7)$ & $309(31,4)$ & $1,30(1,14-1,48)$ & $<0,0001$ \\
\hline
\end{tabular}

(continua) 


\begin{tabular}{|c|c|c|c|c|c|c|}
\hline \multirow[t]{3}{*}{ Fatores de vulnerabilidade } & \multicolumn{4}{|c|}{ Níveis de conhecimento * } & \multirow[t]{3}{*}{ OR ** (IC95\%) } & \multirow[t]{3}{*}{ Valor de $p$} \\
\hline & Total & $\begin{array}{c}\text { Alto } \\
(n=1.370)\end{array}$ & $\begin{array}{c}\text { Médio } \\
(n=1.401)\end{array}$ & $\begin{array}{c}\text { Baixo } \\
(\mathrm{n}=975)\end{array}$ & & \\
\hline & $\%$ & n (\%) & n (\%) & n (\%) & & \\
\hline \multicolumn{7}{|l|}{ Individual } \\
\hline \multicolumn{7}{|l|}{ História de DST nos últimos 12 meses } \\
\hline Sim & 0,8 & $1.368(36,6)$ & $1.395(37,3)$ & $973(26,0)$ & 1,00 & \\
\hline Não & 99,2 & $2(22,2)$ & $5(55,6)$ & $2(22,2)$ & $1,11(0,59-2,07)$ & 0,76 \\
\hline \multicolumn{7}{|c|}{ Sentir-se triste ou deprimido nos últimos 6 meses } \\
\hline Algumas/Muitas vezes & 54,6 & $694(38,1)$ & $685(37,6)$ & $444(24,4)$ & 1,00 & \\
\hline Nunca/Pouco & 45,4 & $674(35,1)$ & $715(37,3)$ & $531(27,7)$ & $1,19(1,06-1,34)$ & 0,004 \\
\hline \multicolumn{7}{|l|}{ Resultado de HIV } \\
\hline Negativo & 88,9 & $1.016(34,3)$ & $1.097(37,1)$ & $846(28,6)$ & 1,00 & \\
\hline Positivo & 11,1 & $141(43,7)$ & $138(42,7)$ & $44(13,6)$ & $2,20(1,81-2,67)$ & $<0,0001$ \\
\hline \multicolumn{7}{|l|}{ Resultado de sífilis } \\
\hline Negativo & 86,1 & $1.027(34,8)$ & $1.102(37,4)$ & $820(27,8)$ & 1,00 & \\
\hline Positivo & 13,9 & $137(40,4)$ & $133(39,2)$ & $69(20,4)$ & $1,08(0,91-1,29)$ & 0,37 \\
\hline \multicolumn{7}{|l|}{ Programática } \\
\hline \multicolumn{7}{|l|}{ Participa de atividade religiosa } \\
\hline Não & 58,2 & $807(37,8)$ & $792(37,0)$ & $539(25,2)$ & 1,00 & \\
\hline Sim & 41,8 & $563(35,0)$ & $608(37,8)$ & $436(27,1)$ & $1,27(1,13-1,43)$ & $<0,0001$ \\
\hline \multicolumn{7}{|c|}{ Participa de atividades em serviços de saúde } \\
\hline Sim & 13,6 & $190(39,1)$ & $196(40,3)$ & $100(20,6)$ & 1,00 & \\
\hline Não & 86,4 & $1.180(36,2)$ & $1.204(36,9)$ & $875(26,9)$ & $1,36(1,15-1,62)$ & 0,0004 \\
\hline \multicolumn{7}{|l|}{ Conhece algum grupo organizado ou ONG } \\
\hline Sim & 26,4 & $457(47,9)$ & $126(13,2)$ & $126(13,2)$ & 1,00 & \\
\hline Não & 73,6 & $913(32,7)$ & $849(30,4)$ & $849(30,4)$ & $2,27(1,98-2,60)$ & $<0,0001$ \\
\hline \multicolumn{7}{|c|}{ Participa de algum grupo organizado ou ONG } \\
\hline Sim & 10,0 & $168(41,4)$ & $62(15,3)$ & $62(15,3)$ & 1,00 & \\
\hline Não & 90,0 & $1.202(36,0)$ & $913(27,3)$ & $913(27,3)$ & $1,71(1,40-2,09)$ & $<0,0001$ \\
\hline \multicolumn{7}{|l|}{ Fez alguma vez o teste para HIV } \\
\hline Sim & 50,5 & $910(46,2)$ & $311(15,8)$ & $311(15,8)$ & 1,00 & \\
\hline Não & 49,5 & $459(25,8)$ & $664(37,4)$ & $664(37,4)$ & $3,67(3,24-4,16)$ & $<0,0001$ \\
\hline \multicolumn{7}{|l|}{ Fez alguma vez o teste para sífilis } \\
\hline Sim & 25,0 & $507(49,1)$ & $137(13,3)$ & $137(13,3)$ & 1,00 & \\
\hline Não & 75,0 & $811(31,5)$ & $791(30,7)$ & $791(30,7)$ & $2,49(2,16-2,87)$ & $<0,0001$ \\
\hline \multicolumn{7}{|c|}{ Recebeu preservativos gratuitos no último mês } \\
\hline Sim/Suficiente & 55,9 & $882(39,9)$ & $485(22,0)$ & $485(22,0)$ & 1,00 & \\
\hline Não recebeu ou recebeu insuficiente & 44,1 & $487(31,8)$ & $489(31,9)$ & $489(31,9)$ & $1,59(1,41-1,79)$ & $<0,0001$ \\
\hline \multicolumn{7}{|c|}{ Recebeu gel lubrificante nos últimos 12 meses } \\
\hline Sim & 14,9 & $295(45,8)$ & $108(16,8)$ & $108(16,8)$ & 1,00 & \\
\hline Não & 85,1 & $1.075(34,7)$ & $866(27,9)$ & $866(27,9)$ & $20,4(1,72-2,41)$ & $<0,0001$ \\
\hline
\end{tabular}

IC95\%: intervalo de 95\% de confiança; ONG: organização não-governamental; OR: odds ratio.

* Excluídos os ignorados;

** OR ponderado de acordo com o tamanho da rede social informada por cada participante e proporção de HSH de cada município pelo total da amostra. 


\section{Tabela 2}

Modelos intermediários e final dos níveis de conhecimento em HIV/AIDS entre os homens que fazem sexo com homens (HSH), Brasil ( $\mathrm{N}=3.746)$.

\begin{tabular}{|c|c|c|c|c|}
\hline \multirow[t]{2}{*}{ Fatores de vulnerabilidade } & \multicolumn{3}{|c|}{ Modelos intermediários } & \multirow{2}{*}{$\begin{array}{c}\text { Modelo final global * } \\
\text { OR (IC95\%) }\end{array}$} \\
\hline & $\begin{array}{c}\text { Social ** } \\
\text { OR (IC95\%) }\end{array}$ & $\begin{array}{l}\text { Individual *** } \\
\text { OR (IC95\%) }\end{array}$ & $\begin{array}{c}\text { Programática \# } \\
\text { OR (IC95\%) }\end{array}$ & \\
\hline \multicolumn{5}{|l|}{ Social } \\
\hline Escolaridade $\leq 8$ anos & $2,06(1,77-2,39)$ & & & $2,11(1,82-2,45)$ \\
\hline Cor da pele não branca & $1,34(1,16-1,56)$ & & & $1,32(1,13-1,53)$ \\
\hline Morar com os pais & $1,40(1,22-1,60)$ & & & \\
\hline Renda individual $<\mathrm{R} \$ 750,00$ & $1,62(1,38-1,91)$ & & & \\
\hline \multicolumn{5}{|l|}{ Critério Brasil de Classificação Econômica } \\
\hline $\mathrm{C}$ & $1,77(1,49-2,11)$ & & & $1,62(1,36-1,92)$ \\
\hline$D-E$ & $2,22(1,79-2,75)$ & & & $2,10(1,71-2,59)$ \\
\hline \multicolumn{5}{|l|}{ Individual } \\
\hline Idade $<25$ anos & & $1,64(1,45-1,87)$ & & $1,78(1,55-2,05)$ \\
\hline Idade da 1ạ relação sexual $\leq 14$ anos & & $1,35(1,19-1,53)$ & & \\
\hline \multicolumn{5}{|l|}{ Orientação sexual } \\
\hline HSH/Homossexual & & $1,26(1,06-1,51)$ & & \\
\hline Bissexual/Outro & & $1,58(1,32-1,89)$ & & \\
\hline Somente um parceiro sexual nos últimos 6 meses & & $1,25(1,09-1,43)$ & & $1,30(1,13-1,50)$ \\
\hline Não sabe a possibilidade de se infectar com o HIV & & $1,57(1.34-1,85)$ & & \\
\hline Esquecer-se de usar preservativos com o uso de álcool e drogas & & $1,32(1,15-1,50)$ & & \\
\hline Não ter relação sexual com ingestão de bebida alcoólica & & $1,57(1,37-1,80)$ & & \\
\hline Ter relação sexual com o uso de droga ilícita & & $1,30(1,12-1,50)$ & & \\
\hline \multicolumn{5}{|l|}{ Programática } \\
\hline Não conhecer algum grupo organizado ou ONG & & & $1,31(1,12-1,53)$ & \\
\hline Nunca ter feito o teste para o HIV & & & $2,94(2,54-3,40)$ & $2,72(2,37-3,13)$ \\
\hline Nunca ter feito o teste para sífilis & & & $1,26(1,07-1,49)$ & \\
\hline Não recebeu ou recebeu quantidade insuficiente de & & & $1,24(1,09-1,40)$ & \\
\hline \multicolumn{5}{|l|}{ preservativos gratuitos no último mês } \\
\hline Não recebeu gel lubrificante nos últimos 12 meses & & & $1,35(1,12-1,62)$ & \\
\hline
\end{tabular}

IC95\%: intervalo de 95\% de confiança; ONG: organização não-governamental; OR: odds ratio.

* Escore do teste do pressuposto do OR proporcional: $\chi^{2}=13,54 ; p=0,06$;

** Escore do teste do pressuposto do OR proporcional: $\chi^{2}=11,47 ; p=0,07$;

*** Escore do teste do pressuposto do OR proporcional: $\chi^{2}=7,35 ; p=0,60$;

\# Escore do teste do pressuposto do OR proporcional: $\chi^{2}=9,63 ; p=0,09$.

Nota: OR proporcional de baixo conhecimento comparando com médio e alto.

com condições socioeconômicas desfavoráveis vem sendo relatado por pesquisadores desde o final da década de 1990 27. Irffi et al. 28 apontam que esse processo pode ter sido influenciado por um desnível significativo no nível de conhecimento sobre HIV/AIDS, desfavorecendo aqueles com as piores condições de educação e classe social. Melhor escolaridade estimula a demanda por conhecimentos específicos sobre a doença, e facilita o entendimento dos riscos de contágio quando as informações são providas por meio da mídia, familiares, ONGs e serviços. Adimora \& Auerbach 29 indicam que as intervenções estruturais que abordam os determinantes sociais da infecção pelo HIV podem estar entre os métodos mais eficientes de prevenção desta infecção, e que as pesquisas devem traçar com clareza os caminhos entre os determinantes sociais e a infecção pelo HIV e desenvolver novas metodologias que possam testar estas intervenções.

Na dimensão de vulnerabilidade individual, os resultados apontam para um baixo conhecimento entre HSH jovens com idades abaixo de 25 anos. É possível que as políticas de prevenção e as campa- 
nhas de massa não estejam atingindo essa população no grau desejado e necessário. Esse dado reforça o preocupante aumento proporcional de 25,2\% para 46,4\% dos casos de AIDS notificados no Brasil entre os HSH na faixa etária de 15 a 24 anos 30 . Estudos realizados por Camargo et al. 31 e Carmargo \& Botelho 32 avaliaram o conhecimento sobre o HIV entre estudantes do Ensino Médio da rede pública. Constataram que o nível era insuficiente para considerá-los cientificamente bem informados, e que falhas ainda existiam no conhecimento sobre transmissão e prevenção ao HIV. Indicaram também que a escola e a televisão foram as duas principais fontes de informação dos adolescentes sobre AIDS e propuseram uma revisão da estratégia de prevenção por meio de multiplicadores, revalorização da família na interlocução e utilização de material informativo próprio para os adolescentes. Da mesma forma, a associação encontrada com a idade da primeira relação sexual abaixo de 14 anos reforça a importância e a necessidade da implantação precoce de uma educação sexual formal na promoção da saúde e bem-estar dos adolescentes, com a participação da escola e da família, de ações preventivas e de campanhas dirigidas aos adolescentes, que abordem conteúdos de qualidade mais abrangentes e esclarecedores sobre as DST e a sexualidade 32. Interações com outros fatores individuais e sociais foram mostrados por Hugo et al. 33 , em que maior risco de ter iniciação sexual precoce foi associado ao sexo masculino, baixo nível socioeconômico, baixa escolaridade, ter pais separados, morar com o parceiro, uso de tabaco e drogas, e não uso da camisinha na última relação. Com relação ao fator individual orientação sexual, o maior risco encontrado com o baixo conhecimento foi entre os homens que se autodeclararam bissexuais. Segundo Greco et al. ${ }^{34}$, as situações de risco para HIV foram mais frequentes entre os homens que relataram atividade sexual com homens e mulheres. Os comportamentos sexuais e de proteção dos bissexuais diferem conforme gênero e estabilidade da parceria, havendo menor proteção com parceiras fixas mulheres. Indicam ainda que a maior vulnerabilidade dos bissexuais à infecção pelo HIV em comparação aos homossexuais pode estar relacionada à construção social da masculinidade, uma vez que parte da população bissexual ainda recorre a estratégias de encobrimento, para evitar discriminação, agressão verbal e violência física, isolando-se em seu ambiente microssocial, o que dificulta as ações preventivas a este grupo específico.

Dentre os fatores indicadores de vulnerabilidade individual, mostrou-se preocupante a associação encontrada entre não saber a sua chance de se infectar com o vírus do HIV e o baixo conhecimento. A falta de percepção de risco ou a negação da vulnerabilidade ao HIV e às DST pode trazer um reflexo negativo na adoção de práticas sexuais seguras e na busca da testagem para o HIV 35. É fundamental avaliar e reformular as estratégias de disponibilização de informações sobre o HIV/AIDS, de forma que atinja integralmente essa população. Sendo assim, esforços concentrados específicos devem ser realizados para aumentar a sensibilidade para as necessidades de saúde dos HSH, melhorando o acesso aos serviços de saúde e a participação de organizações da sociedade civil. Além disso, é possível ainda que o grande avanço nos últimos anos no controle do HIV/AIDS, com maior sobrevida e melhor qualidade de vida, possa prejudicar a percepção do risco entre os $\mathrm{HSH}$ 36,37. Além disso, segundo estudo de McDaid \& Hart 38 , HSH que tiveram mais contato com atividades de prevenção do HIV foram aqueles considerados em maior risco, isto é, apresentaram resultado positivo para o HIV ou outra DST, relataram ter tido dez ou mais parceiros ou história anterior de teste para o HIV ou outra DST nos últimos 12 meses. De forma semelhante, encontramos neste estudo que ter somente um parceiro sexual aumenta a chance de registrar baixo conhecimento em HIV/AIDS, o que pode conduzir a não percepção do risco e, consequentemente, a não necessidade da busca de algum serviço que o oriente a respeito. Resultado similar também foi encontrado no trabalho realizado por Deb et al. 15. Contudo, esse fato se torna preocupante quando analisamos os resultados de Rocha et al. 4 , que encontraram um elevado risco de prática do sexo anal receptivo desprotegido entre os $\mathrm{HSH}$ que vivem com um parceiro masculino ou têm um parceiro estável, dos quais uma grande proporção destes também relatou relações sexuais com um parceiro casual ou comercial ao mesmo tempo.

Ainda são desafiadores o entendimento e as intervenções para a prevenção do HIV no âmbito do uso de álcool e/ou drogas. Encontramos o baixo conhecimento associado tanto com HSH que relataram já ter se esquecido de usar preservativos com o uso de álcool e/ou drogas quanto aqueles que não tiveram relação sexual com ingestão de bebidas alcoólicas. Mustanski et al. ${ }^{39}$ relataram que ainda são inconsistentes os modelos teóricos propostos para explicar por que o uso do álcool pode estar associado à falta do uso de preservativos. Estudos têm demonstrado que apesar do conhecimento sobre medidas preventivas, a utilização do preservativo é baixa em grupos mais vulneráveis, como os HSH e 
adolescentes, especialmente sob a influência do álcool e/ou de outras substâncias psicoativas. O álcool é comumente usado como um desinibidor, facilitador na abordagem do sexo, na recreação, na socialização, e os bares e boates têm emergido como locais atrativos aos jovens, favorecendo o aumento do consumo do álcool e a iniciação sexual precoce. Contudo, esses estudos destacam a necessidade de se analisar esse ponto com base nos aspectos socioculturais e individuais, a fim de se planejar as intervenções apropriadas 40 .

Os resultados da análise dos fatores indicadores de vulnerabilidade programática indicam potenciais falhas na estratégia das políticas de saúde pública e na participação da sociedade civil (ONGs e demais parcerias) junto à população de HSH. A associação entre não conhecer algum grupo organizado ou ONG, não receber preservativos gratuitamente, receber número insuficiente de preservativos ou não receber gel lubrificante e o baixo conhecimento aponta para a falta de acessibilidade às informações de promoção da prevenção. Provavelmente, trata-se de indivíduos que parecem não terem sido atingidos pela mídia, pelas campanhas sobre prevenção primária e nem foram orientados sobre a distribuição gratuita dos insumos pelos serviços de saúde e organizações da sociedade civil parceiras 41 . A dificuldade de acesso ou o afastamento desse grupo das ações de cuidado e da promoção de saúde pode estar relacionada ao constrangimento de exposição devido as suas práticas sexuais ainda discriminadas, combinadas com o preconceito, a homofobia e o estigma da doença 35 . Esses resultados indicam urgente avaliação e aprimoramento do acesso e acolhimento dessa população aos serviços básicos e especializados de saúde, e a adequação estrutural das estratégias de prevenção, com abordagens e métodos que considerem contextos mais amplos e equipes multiprofissionais capacitadas, que atuem com as especificidades desse subgrupo. Um estudo realizado por Colosio et al. 42 mostrou que os HSH participantes de grupos operativos foram favoráveis à mudança em favor da adoção da prática de sexo mais seguro, e que este método de intervenção preventiva permite lidar com as três dimensões da vulnerabilidade (individual, social e programática). Charania et al. $43 \mathrm{em}$ uma meta-análise relataram que as intervenções que aumentam a disponibilidade ou acessibilidade aos preservativos mostraramse um meio eficaz para incrementar o uso de preservativos na prevenção de HIV/DST. Programas de promoção do sexo seguro podem ser mais bem-sucedidos se disponibilizarem preservativos com mais frequência em locais como bares, cafés, saunas, banheiros públicos, bordéis e hotéis, para que estejam prontamente disponíveis no momento em que forem necessários 44.

Nunca ter feito o teste para HIV mostrou-se como um dos fatores mais fortemente associados ao baixo conhecimento. Esse indicador de vulnerabilidade programática pode ser entendido como um marcador (proxy) do acesso à informação, educação sexual e comunicação em relação ao HIV nessa população. Liu et al. 16 relataram que os HSH testados para o HIV há mais de um ano tinham significativamente um maior nível de conhecimento do que aqueles que nunca foram testados. Desde 2010, o Brasil vem incrementando a sua estratégia de testagem para o HIV, visando à ampliação da cobertura de diagnóstico no país, principalmente a de segmentos mais vulneráveis da população 39 . Sendo assim, ressaltamos que se faz necessário um maior esforço e articulação dos gestores públicos para que a cobertura de diagnóstico amplie rapidamente para a rede de atenção básica, e que haja um maior reconhecimento e participação da sociedade civil na promoção, divulgação e sensibilização da população para a realização do teste rápido do HIV e sífilis. Do ponto de vista programático, os indivíduos podem certamente obter benefícios de acesso a cuidados integrados, com ênfase na prevenção.

Algumas considerações sobre este trabalho devem ser mencionadas. Inicialmente, trata-se de um estudo de corte transversal, que embora aponte possíveis fatores de riscos para um determinado evento quando uma associação é encontrada, apresenta uma limitação quanto ao estabelecimento de relações de causalidade. Segundo, a amostra estudada foi composta pela agregação de dez amostras independentes dos municípios selecionados, e sabe-se que diferenças existem entre os municípios e, portanto, devem ser investigadas. Além disso, considerando que o estudo principal foi, inicialmente, delineado para estimar a infecção pelo HIV, mas não necessariamente para avaliar o conhecimento, os resultados deste trabalho podem, potencialmente, apresentar limitado poder estatístico para detectar associações de interesse. Finalmente, é importante ressaltar que embora a técnica amostral RDS seja reconhecida pela sua capacidade de alcançar populações de difícil acesso, atingindo setores da população muitas vezes não acessíveis por outras metodologias, ela ainda está sujeita a um tipo de viés sistemático, pois as cadeias de recrutamento refletem padrões de afiliação que tendem a ocorrer entre pessoas que se assemelham em níveis de educação, renda, etnia e de interesses. Sendo assim, é 
provável que participantes com nível de conhecimento maior ou menor possam ter convidado pessoas com conhecimento similar. Tal efeito potencialmente afeta as estimativas de prevalência, mas não necessariamente a avaliação dos fatores associados. Em conclusão, os resultados dos fatores de vulnerabilidade com o baixo conhecimento ressaltaram uma questão relevante que é o papel das desigualdades sociais na trajetória da epidemia, o que exige uma maior conscientização e mobilização coletiva para uma mudança impactante com relação à vulnerabilidade ao HIV nessa população. Além disso, trazem novos desafios para a área da saúde, para seus gestores e demais parceiros, no direcionamento de ações preventivas para além do comportamento individual, baseado na realidade epidemiológica e contextual das diversas comunidades (fatores sociais e culturais), com ampliação da programação para aqueles de maior risco, como os jovens em condições socioeconômicas mais desfavoráveis. Os fatores de vulnerabilidade programática têm elementos essenciais que podem reduzir os fatores de vulnerabilidade individual e social, por meio de intervenções de nível estrutural que promovam a melhoria do conhecimento em HIV/AIDS, a autopercepção de risco e comportamentos em saúde, como o uso consistente dos preservativos e a realização da testagem, importante componente de prevenção do HIV. Vale ressaltar o fundamental papel da sociedade civil, das ONGs, na ocupação de espaços de controle social, na promoção e garantia dos direitos humanos, na eliminação de barreiras legais que comprometem o acesso aos serviços de saúde, no monitoramento e avaliação dos programas de prevenção. Da mesma forma, é fundamental estabelecer uma vigilância permanente de acompanhamento e avaliação do conhecimento e das informações relacionadas ao HIV nessa população de estudo, que permita evidenciar o impacto dos investimentos e transformações ocorridas ao longo do tempo nas três dimensões: social, individual e programática. Espera-se que os resultados deste estudo possam subsidiar pesquisas futuras no monitoramento dos níveis de conhecimento em HIV/AIDS e contribuir na formulação de políticas de saúde, preventivas e de intervenção que promovam a redução das vulnerabilidades dessa população no Brasil.

\section{Colaboradores}

R. R. F. M. Gomes participou de todas as etapas do artigo, revisão da literatura, análises e interpretação dos dados, redação e revisão crítica. M. G. B. Ceccato e M. D. C. Guimarães contribuíram na redação e revisão crítica do artigo. L. R. F. S. Kerr colaborou na revisão crítica do artigo.

\section{Agradecimentos}

Este trabalho foi financiado pelo Departamento de DST, Aids e Hepatites Virais, Ministério da Saúde, por intermédio de uma colaboração entre o Governo Brasileiro e o Escritório das Nações Unidas para Drogas e Crime (UNODC) (projeto AD/BRA/ 03/H34). 


\section{Referências}

1. Johnson AS, Hall HI, Hu X, Lansky A, Holtgrave DR, Mermin J. Trends in diagnoses of HIV infection in the United States, 2002-2011. JAMA 2014; 312:432-4.

2. Beyrer C, Baral SD, van Griensven F, Goodreau SM, Chariyalertsa S, Wirtz AL, et al. Global epidemiology of HIV infection in men who have sex with men. Lancet 2012; 380: 367-77.

3. Kerr LR, Mota RS, Kendall C, Pinho AA, Mello MB, Guimarães MD, et al. HIV among MSM in a large middle-income country. AIDS 2013; 27:427-35.

4. Rocha GM, Kerr LRFS, Brito AM, Dourado I, Guimarães MDC. Unprotected receptive anal intercourse among men who have sex with men in Brazil. AIDS Behav 2013; 17:1288-95.

5. Gomes RRFM, Batista JR, Ceccato MGB, Kerr LRFS, Guimarães MDC. HIV/AIDS knowledge among men who have sex with men in Brazil: applying the item response theory. Rev Saúde Pública 2014; 48:206-15.

6. Joint United Nations Programme on HIV/ AIDS. Sexual behavioural change for HIV: where have theories taken us? Geneva: Joint United Nations Programme on HIV/AIDS; 1999.

7. Wellings K, Collumbien M, Slaymaker E, Singh S, Hodges Z, Patel D, et al. Sexual behaviour in context: a global perspective. Lancet 2006; 368:1706-28.

8. Mann J, Tarantola DJM, Netter TW. A Aids no mundo. Rio de Janeiro: Editora Relume-Dumará/ Associação Brasileira Interdisciplinar de AIDS/Instituto de Medicina Social, Universidade do Estado do Rio de Janeiro; 1993.

9. Mann J, Tarantola DJM, editors. AIDS in the world II: global dimensions, social roots, and responses. New York: Oxford University Press; 1996.

10. Garcia S, Souza FM. Vulnerabilidades ao HIV/ aids no contexto brasileiro: iniquidades de gênero, raça e geração. Saúde Soc 2010; 19 Suppl 2:9-20.

11. Schaurich D, Freitas HMB. O referencial de vulnerabilidade ao HIV/AIDS aplicado às famílias: um exercício reflexivo. Rev Esc Enferm USP 2011; 45:989-95.

12. Sánchez AIM, Bertolozzi MR. Pode o conceito de vulnerabilidade apoiar a construção do conhecimento em saúde coletiva? Ciênc Saúde Coletiva 2007; 12:319-24.

13. Programa Nacional de DST e Aids, Secretaria de Vigilância Saúde, Ministério da Saúde. Pesquisa de conhecimento, atitudes e práticas na população brasileira de 15 a 54 anos, 2004. Brasília: Ministério da Saúde; 2005.

14. Departamento de DST, Aids e Hepatites Virais, Secretaria de Vigilância em Saúde, Ministério da Saúde. PCAP-2008: pesquisa de conhecimentos, atitudes e práticas na população brasileira de 15 a 64 anos, 2008. Brasília: Ministério da Saúde; 2011.
15. Deb S, Dutta1 S, Dasgupta A, Biswas B. Sexual practice and perception of HIV/AIDS amongst men who have sex with men in Kolkata. Indian J Community Med 2009; 34:206-11.

16. Liu S, Wang K, Yao S, Guo X, Liu Y, Wang B. Knowledge and risk behaviors related to HIV/ AIDS and their association with information resource among men who have sex with men in Heilongjiang province, China. BMC Public Health 2010; 10:250.

17. Knox J, Sandfort T, Yi H, Reddy V, Maimane S. Social vulnerability and HIV testing among South African men who have sex with men (MSM). Int J STD AIDS 2011; 22:709-13.

18. Wagenaar BH, Sullivan PS, Stephenson R. HIV knowledge and associated factor among internet-using men who have sex with men (MSM) in South Africa and the United States. PLoS One 2012; 7:e32915.

19. Pando MA, Balan I, Marone R, Dolezal C, Barreda V, Dieguez AC, et al. HIV knowledge and beliefs among men who have sex with men (MSM) in Buenos Aires, Argentina. AIDS Behav 2013; 17:1305-12.

20. Heckathorn DD. Respondent-driven sampling: a new approach to the study of hidden populations. Soc Probl 1997; 44:174-99.

21. Joint United Nations Programme on HIV/ AIDS. United Nations General Assembly Special Session on HIV/AIDS. Monitoring the declaration of commitment on HIV/AIDS: guidelines on constructions of core indicators. Geneva: Joint United Nations Programme on HIV/AIDS; 2008.

22. Szwarcwald CL, Souza Júnior PRB, Damacena GN, Barbosa Júnior A, Kendall C. Analysis of data collected by RDS among sex workers in 10 Brazilian cities, 2009: estimation of the prevalence of HIV, variance, and design effect. J Acquir Immune Defic Syndr 2011; 57 Suppl 3:S129-35.

23. Bender R, Grouven U. Ordinal logistic regression in medical research. J R Coll Physicians Lond 1997; 31:546-51.

24. Abreu MNS, Siqueira AL, Caiaffa WT. Ordinal logistic regression in epidemiological studies. Rev Saúde Pública 2009; 43:183-94.

25. Pasquali L, Primi R. Fundamentos da teoria da resposta ao item-TRI. Aval Psicol 2003; 2: 99-110.

26. Organização Internacional do Trabalho. Recomendação sobre o HIV e a AIDS e o mundo do trabalho: adoção OIT 2010. http://www.oi tbrasil.org.br/content/recomenda\%C3\%A7\% C3\%A3o-sobre-o-hiv-e-aids-e-o-mundo-dotrabalho (acessado em 19/Jul/2015).

27. Fonseca MGP, Travassos C, Bastos FI, Silva NVS. Szwarcwald CL. Distribuição social da AIDS no Brasil, segundo participação no mercado de trabalho, ocupação e status sócio-econômico dos casos de 1987 a 1998. Cad Saúde Pública 2003; 19:1351-63. 
28. Irffi G, Soares RB, Desouza AS. Fatores socioeconômicos, demográficos, regionais e comportamentais que influenciam no conhecimento sobre HIV/AIDS. Economia 2010; 11:333-56.

29. Adimora AA, Auerbach JD. Structural interventions for HIV prevention in the United States. J Acquir Immune Defic Syndr 2010; 55 Suppl 2:S132-5.

30. Aids no Brasil: epidemia concentrada e estabilizada em populações de maior vulnerabilidade Boletim Epidemiológico 2012; 43:8-10.

31. Camargo BV, Barbará A, Bertoldo RB. Concepção pragmática e científica dos adolescentes sobre a Aids. Psicol Estud 2007; 12:277-84.

32. Camargo BV, Botelho LJ. Aids, sexualidade e atitudes sobre a proteção contra o HIV. Rev Saúde Pública 2007; 41:61-8.

33. Hugo TDO, Maier VT, Jansen K, Rodrigues CEG, Cruzeiro ALS, Ores LC, et al. Fatores associados à idade da primeira relação sexual em jovens: estudo de base populacional. Cad Saúde Pública 2011; 27:2207-14.

34. Greco M, Silva AP, Merchán-Hamann E, Jeronymo ML, Andrade JC, Greco DB. Diferenças nas situações de risco para HIV de homens bissexuais em suas relações com homens e mulheres. Rev Saúde Pública 2007; 41 Suppl 2:109-17.

35. Programa Nacional de DST e Aids, Secretaria de Vigilância em Saúde, Ministério da Saúde. Plano Nacional de Enfrentamento da Epidemia de Aids e das DST entre Gays, HSH e Travestis. Brasília: Ministério da Saúde; 2007.

36. Elford J, Bolding G, Sheerr L. High-risk sexual behavior increases among London gay men between 1998 and 2001: what is the role of HIV optimism? AIDS 2002;16:1537-44.

37. Lima FSS, Silva MJG, Godoi AMM, Merchan -Hamann E. Homens que fazem sexo com homens: uso dos serviços de saúde para prevenção/controle de HIV/AIDS em Brasília-DF. Comun Ciênc Saúde 2008; 19:25-34.
38. McDaid LM, Hart GJ. Contact with HIV prevention services highest in gay and bisexual men at greatest risk: cross-sectional survey in Scotland. BMC Public Health 2010; 10:798.

39. Mustanski BS, Newcomb ME, DuBois SN, Garcia SC, Grov C. HIV in young men who have sex with men: a review of epidemiology, risk and protective factors and interventions. J Sex Res 2011; 48:218-53.

40. World Health Organization. Alcohol use and sexual risk behaviour: a cross-cultural study in eight countries. Geneva: World Health Otrganization; 2005.

41. Departamento de DST, Aids e Hepatites Virais, Secretaria de Vigilância em Saúde, Ministério da Saúde. Relatório de progresso da resposta brasileira ao HIV/AIDS (2010-2011). Brasília: Ministério da Saúde; 2012.

42. Colosio R, Fernandes MIA, Bergamaschi DP, Scarcelli IR, Lopes IC, Hearst N. Prevenção de infecção pelo HIV por intermédio da utilização do grupo operativo entre homens que fazem sexo com homens, São Paulo, Brasil. Cad Saúde Pública 2007; 23:949-59.

43. Charania MR, Crepaz N, Guenther-Gray C, Henny K, Liau A, Willis LA, et al. Efficacy of structural-level condom distribution interventions: a meta-analysis of U.S. and international studies, 1998-2007. AIDS Behav 2011; 15:1283-97.

44. Colby DJ. HIV knowledge and risk factors among men who have sex with men in Ho Chi Minh City, Vietnam. J Acquir Immune Defic Syndr 2003; 32:80-5. 


\section{Abstract}

This cross-sectional study analyzed social vulnerability, individual, and programmatic factors associated with low knowledge on HIV/AIDS among men who have sex with men (MSM). Respondent Driven Sampling (RDS) was used in 10 Brazilian cities. Knowledge scores on HIV/AIDS were estimated by Item Response Theory and categorized in three levels: high, medium, and low knowledge. Ordinal logistic regression was used for the analysis. Of the 3,746 MSM, 36.6\%, 37.4\%, and 26\% showed high, medium, and low knowledge, respectively. The following were associated with low knowledge in the final model: schooling $\leq 8$ years, non-white skin color, economic classes $C$ and D-E, age $<25$ years, having only one sex partner, and no previous HIV test. It is essential to improve knowledge on HIV/AIDS among young MSM with low socioeconomic status. Interventions focused on programmatic vulnerability factors can help reduce individual and social risk.

HIV; Risk Groups; Male Homosexuality; Health Knowledge, Attitudes, Practice; Health Vulnerability

\section{Resumen}

Este estudio transversal analizó los factores de vulnerabilidad social, individual y programática, asociados al bajo conocimiento en VIH/SIDA entre hombres que practican sexo con hombres (HSH). La técnica de muestra Respondent Driven Sampling (RDS) se utilizó en diez ciudades brasileñas. Los marcadores de conocimiento en VIH/SIDA se estimaron por la Teoría de Respuesta al Ítem y se categorizaron en tres niveles: alto, medio y bajo conocimiento. El modelo de regresión logística ordinal se utilizó para el análisis. Entre los 3.746 HSH se halló que un 36,6\%, 37,4\% y 26\% tenían alto, medio y bajo conocimiento, respectivamente. En el modelo final, presentaron asociación con bajo conocimiento: escolaridad $\leq 8$ años, color de piel no blanca, criterio de clase económica $C$ y D-E, edad $<25$ años, tener solamente un compañero sexual y nunca haberse hecho el test anti-HIV. Es fundamental mejorar el nivel de conocimiento sobre VIH/SIDA entre los jóvenes HSH, con condiciones socioeconómicas desfavorables. Intervenciones enfocadas en los factores de vulnerabilidad programática pueden contribuir a la reducción de los factores individual y social.

VIH; Grupos Vulnerables; Homosexualidad Masculina; Conocimientos, Actitudes y Práctica en Salud; Vulnerabilidad en Salud
Recebido em 06/Ago/2015

Versão final reapresentada em 20/Mai/2016

Aprovado em 13/Dez/2016 